\title{
Luminescence properties of hexagonal boron nitride powders probed by deep UV photoluminescence spectroscopy
}

\author{
Nikesh Maharjan ${ }^{1}\left[\right.$ (]) Mim Lal Nakarmi ${ }^{1}$
}

Received: 25 February 2021 / Accepted: 8 April 2021 / Published online: 12 May 2021

(c) The Author(s), under exclusive licence to The Materials Research Society 2021

\begin{abstract}
Deep ultraviolet (UV) photoluminescence (PL) spectroscopy was employed to study the luminescence properties of hexagonal boron nitride (h-BN) crystal powders after annealing the samples at different temperatures in the range of $100-900{ }^{\circ} \mathrm{C}$ for $1 \mathrm{~h}$ in ambient air. The PL spectrum from the h-BN powder samples annealed around $700{ }^{\circ} \mathrm{C}$ showed strong luminescence intensity at $5.49 \mathrm{eV}$ along with enhanced phonon-assisted band-edge emission at $5.90 \mathrm{eV}$. Additionally, it revealed sharp atomic-like emission lines in UV region at 4.10, 4.12, 4.14, and $4.16 \mathrm{eV}$ with line widths less than $1 \mathrm{~nm}$ from the annealed samples which were not present in the unannealed samples. Power- and temperature-dependent PL measurements of the sharp atomic-like emission lines exhibited robust nature of the energy peak positions. Based on the theoretical reports, the sharp emission lines could be carbon-related defects.
\end{abstract}

\section{Introduction}

Inspired by the demonstration of lasing action at wavelength $\sim 215 \mathrm{~nm}$ by electron beam excitation from a bulk hexagonal boron nitride (h-BN) crystal in 2004, it has attracted much interest for its potential applications in deep UV photonics [1]. Luminescence properties of bulk and epilayer h-BN samples have been reported with strong deep UV band-edge-related emissions in deep UV region by using photoluminescence (PL) or cathodoluminescence (CL) [1-4]. Despite the strong UV luminescence, h-BN has been found to be an indirect bandgap semiconductor with energy bandgap $\sim 6 \mathrm{eV}$ with a valence band maximum and conduction band minimum at the $\mathrm{K}$ and $\mathrm{M}$ points of the first Brillouin zone, respectively [5]. The band-edge emissions are produced via phonon-assisted electronic transitions. The strong emission in deep UV region can be used for general purpose lighting, surface disinfection, and sterilization $[6,7]$. There is a huge demand of portable deep UV light sources in germicidal range for disinfection due to COVID-19 pandemic. h-BN is also a promising candidate for single-photon sources [8]. Single-photon emissions have

Nikesh Maharjan

nmaharjan@gradcenter.cuny.edu

1 Department of Physics, Brooklyn College and the Graduate Center of the City University of New York, Brooklyn, NY 11210, USA been reported in the near-infrared, visible, and UV regions from defect states of h-BN samples [9-13]. Although optical properties of h-BN are revealed quickly using bulk and thin-film h-BN samples, optical studies of h-BN in powder form are rare [14]. High-purity nanopowders can be used for lasing gain medium [15]. More works on h-BN powder especially optical studies are needed for understanding its physical properties and applications in optical devices.

In this paper, we report the optical studies of h-BN crystal powders using a deep UV PL spectroscopy. We observed strong PL emission peak at $5.49 \mathrm{eV}$ and phonon-assisted band-edge emission peak at $5.89 \mathrm{eV}$ from the h-BN powder samples annealed around $700{ }^{\circ} \mathrm{C}$ for $1 \mathrm{~h}$ in ambient air. Additionally, the samples also exhibited sharp atomic-like emission lines at 4.10, 4.12, 4.14, and $4.16 \mathrm{eV}$ along with their phonon replicas. We also studied the nature of these emission lines from power- and temperature-dependent PL measurements.

\section{Material and methods}

The h-BN powder samples studied in this work were purchased from Alfa Aesar (99.5\% purity). Visually, the h-BN samples appear white powder with particle size in the order of few microns. Luminescence properties of the annealed samples were studied by employing a deep UV PL spectroscopy system. The PL system used in this study has a 
Coherent Mira900 ultrafast Ti:sapphire laser with pulse width of $\sim 100 \mathrm{fs}$ and repetition rate of $76 \mathrm{MHz}$. For the above bandgap photoexcitation of the h-BN samples, higher harmonics are generated by coupling the Ti:sapphire laser with third harmonic generator and then fourth harmonic generator that produces laser outputs at 266 and $200 \mathrm{~nm}$ with average powers of 100 and $2 \mathrm{~mW}$, respectively. The fourth harmonic laser was used for photoexcitation in all PL measurements in this work. The detection system consists of a Princeton $750 \mathrm{~mm}$ monochromator with a photomultiplier tube. A copper sample holder with fused silica $\left(\mathrm{SiO}_{2}\right)$ window was built for PL measurements of the powder samples. The sample holder was then mounted to a Janis cryostat with a Lakeshore temperature controller.

\section{Results and discussion}

Low-temperature PL measurements were performed at $8 \mathrm{~K}$ for all samples. The PL measurement of unannealed sample was first carried out for reference. Same powder sample was then annealed at different temperatures ranging from 100 to $900{ }^{\circ} \mathrm{C}$ for $1 \mathrm{~h}$ in ambient air. The PL spectra were measured after each sequential annealing. Figure 1 shows the representative PL spectra measured after annealing the sample at 500, 700, and $900{ }^{\circ} \mathrm{C}$ including the reference. In all spectra, the dominant PL emission peak is at around $5.49 \mathrm{eV}$. There is no significant difference in the PL intensity

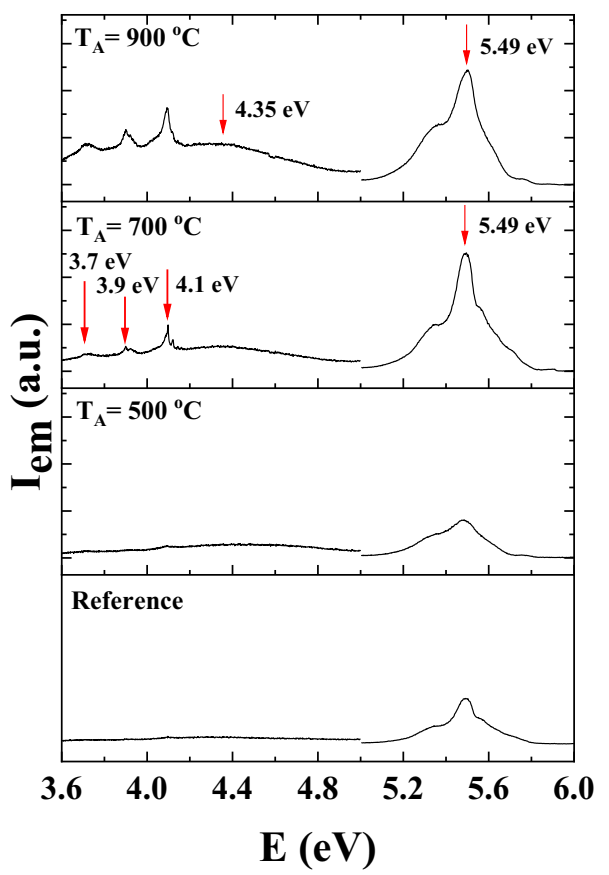

Fig. 1 Low-temperature $(8 \mathrm{~K}) \mathrm{PL}$ spectra of h-BN powder sample after sequentially annealing at different annealing temperatures $\left(T_{\mathrm{A}}\right)$. The data points below $5 \mathrm{eV}$ are magnified 5 times for annealing temperatures up to $500{ }^{\circ} \mathrm{C}$ compared to the reference. The maximum PL intensity is observed after annealing the sample around $700{ }^{\circ} \mathrm{C}$. More other peaks also became pronounced. Enhanced crystalline and optical quality of zinc oxide powders was also observed by annealing [16]. An interesting feature with a sharp emission peak at $4.10 \mathrm{eV}$, and faint peaks at 3.90 and $3.70 \mathrm{eV}$ can be also seen in the PL spectrum of the sample annealed at $700{ }^{\circ} \mathrm{C}$. The data points for the band below $5 \mathrm{eV}$ are magnified five times to see these peaks clearly. Further increasing the annealing temperature decreases the intensity of the dominant peak at $5.49 \mathrm{eV}$ and the sharp emission line at $4.10 \mathrm{eV}$ becomes broader as shown in the PL spectrum measured after annealing around $900{ }^{\circ} \mathrm{C}$. Additionally, the background intensity is increased with peak around $4.35 \mathrm{eV}$ that could be due to impurity transitions.

We also performed the PL measurements after annealing h-BN samples directly to a particular temperature and found similar results to those from the sample annealed sequentially. Figure 2 shows the PL spectrum of the h-BN sample after annealing directly at temperature around $700{ }^{\circ} \mathrm{C}$ for $1 \mathrm{~h}$ in ambient air. The spectrum has been plotted in the log scale to see all the features. All the energy peaks marked are explained as follows and the emission peaks are assigned based on the PL results from bulk h-BN samples. The highest band-edge emission peak observed at around $5.89 \mathrm{eV}$ is assigned to the acoustic phonon-assisted band-edge transition [5]. In the PL spectrum of bulk h-BN samples, they normally have optical phonon-assisted band-edge emission peak around $5.78 \mathrm{eV}$ that is not clearly resolved in the powder sample. However, there is a small shoulder at around $5.71 \mathrm{eV}$. PL emission of h-BN normally has multiple peaks around $5.5 \mathrm{eV}$ whose origin is believed to be due to stacking

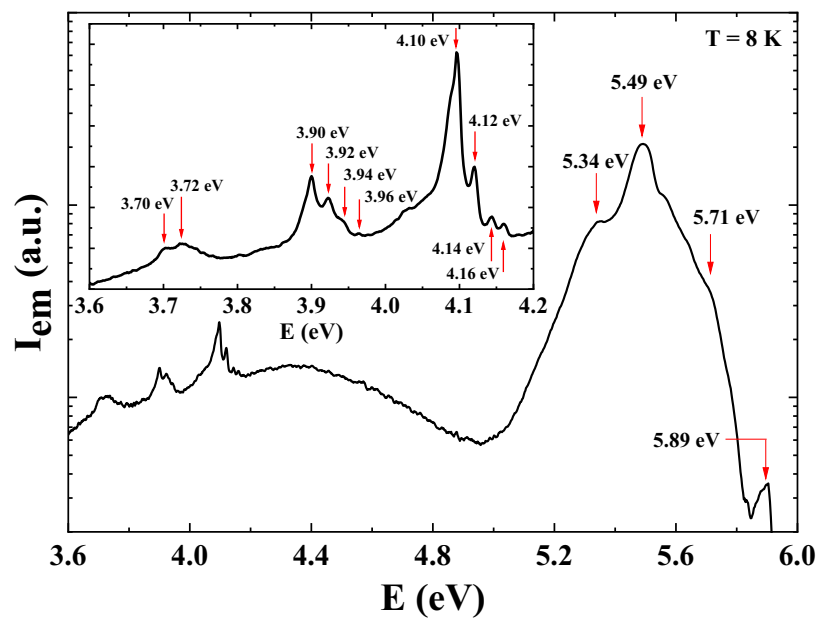

Fig. 2 Low-temperature ( $8 \mathrm{~K}$ ) PL spectrum of h-BN powder sample annealed around $700{ }^{\circ} \mathrm{C}$ for $1 \mathrm{~h}$. Inset: replotted spectrum in the range from 3.6 to $4.2 \mathrm{eV}$ 
faults [17]. We can also see peaks at $5.34 \mathrm{eV}$. Based on the results from first-principles calculations, the emission peaks at $5.34 \mathrm{eV}$ could be due to electronic transition from oxygen replacing nitrogen $\left(\mathrm{O}_{\mathrm{N}}\right)$ to the valence band [18].

In order to see the sharp emission lines clearly, the spectrum is replotted in the range from 3.6 to $4.2 \mathrm{eV}$ and shown in the inset of Fig. 2. The major peak at $4.10 \mathrm{eV}$ has line width about $5 \mathrm{meV}(0.3 \mathrm{~nm})$ as in atomic-like emissions. The peaks at 3.90 and $3.70 \mathrm{eV}$ are assigned to $1 \mathrm{LO}$ and $2 \mathrm{LO}$ phonon replicas of the $4.10 \mathrm{eV}$ line, respectively, as the LO phonon energy in h-BN is about $200 \mathrm{meV}$ [19]. The feature of $4.10 \mathrm{eV}$ line with phonon replica was also reported as single-photon emission from defect points on bulk h-BN [13]. In this sample, along with the dominant sharp emission line at $4.10 \mathrm{eV}$, additional sharp emission peaks can be clearly seen at $4.12,4.14$, and $4.16 \mathrm{eV}$ in the spectrum. We can also see their 1LO phonon above the $1 \mathrm{LO}$ phonon replica of the 4.10 line (i.e., above $3.90 \mathrm{eV}$ ). In most of the reports from bulk samples, only the emission line at 4.10 and its phonon replicas are reported. With careful observation of the phonon replicas, it is also evident that the rate of decreased intensity of the phonon replicas of $4.10 \mathrm{eV}$ line is faster than that of $4.12 \mathrm{eV}$. Thus, we believe the additional peaks are unrelated to the $4.10 \mathrm{eV}$ line. It is worth to mention that these sharp emissions are not just blinks. They are very stable and repeatable. Observation of these additional emission lines at $4.12,4.14$, and $4.16 \mathrm{eV}$ was reported from the carbon-doped h-BN samples [20]. The h-BN powder sample could have more carbon content than in the bulk samples.

In order to understand the nature of the PL emissions from the h-BN powders, we performed power- and temperature-dependent PL measurements of the samples annealed around $700{ }^{\circ} \mathrm{C}$. Figure 3 shows the power-dependent PL measurement performed at $8 \mathrm{~K}$. Average power of the excitation laser was varied from 0.1 to $1 \mathrm{~mW}$. The power range was limited by our system. Intensity of the dominant emission peak at $5.49 \mathrm{eV}$ increases with increasing excitation laser power due to the increased concentrations of electron and hole. Intensity of the $5.49 \mathrm{eV}$ peak is increased by an order when the laser power is changed in that range. It is interesting to note that there is no shift of position of energy peak at $5.49 \mathrm{eV}$ with increasing the excitation power. It indicates the electronic transition related to the emission peak is not donor-acceptor pair transitions, but it is most likely due to the transition from a deep donor to the valence band [21]. The intensity of the $4.10 \mathrm{eV}$ line did not increase at the same rate of the band-edge emission peak with increasing excitation power. Clearly, there is increase of the intensity of the $4.10 \mathrm{eV}$ peak when the laser power was changed from 0.1 to $0.5 \mathrm{~mW}$. But when it was increased to $1 \mathrm{~mW}$ there was almost no change showing saturation. This could be due to the short lifetime of phonon-assisted transition which is in the order of around 200 ps [22]. In the competitive

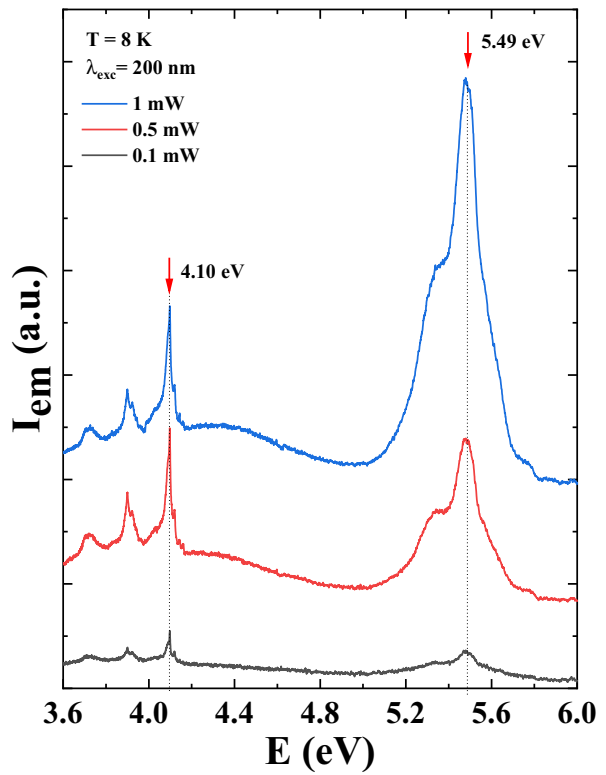

Fig. 3 Power-dependent PL spectra of h-BN powder sample measured at $8 \mathrm{~K}$

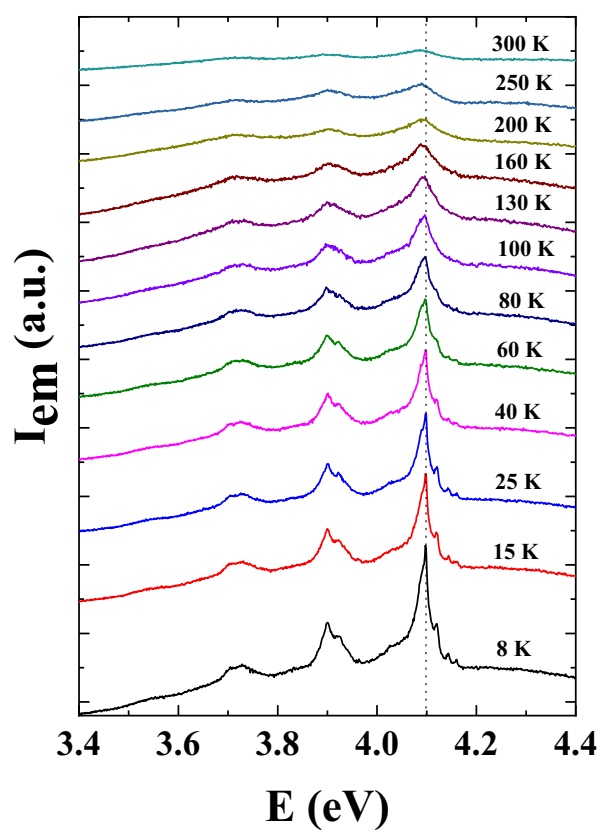

Fig. 4 Temperature-dependent PL spectra of the sharp emission lines in UV region from annealed h-BN powder sample measured by varying the sample temperature from 8 to $300 \mathrm{~K}$

recombination process, band-edge transitions thus dominated compared to the transition related to $4.10 \mathrm{eV}$. It is also interesting to note that there is no indication of energy peak position change while increasing the excitation laser power.

Figure 4 shows the temperature-dependent PL spectra measured by varying the sample temperature from 8 to 
$300 \mathrm{~K}$. As the temperature is increased, the intensity of the sharp emission lines decreased that could be due to the thermal quenching. Additionally, the emission line spectrum became broader with increasing temperature. The sharp feature slowly disappears above $50 \mathrm{~K}$. However, the energy peak position did not shift with changing temperature too. Based on the power- and temperature-dependent PL, results showed that the energy peak position of the sharp emission lines at $4.10 \mathrm{eV}$ are independent of the carrier concentration and the temperature. Thus, we can conclude that the transition levels related to the sharp emission line in UV region from $\mathrm{h}-\mathrm{BN}$ have a robust nature.

The robust nature of the sharp emission lines indicates that the emissions are related to highly localized defect states in the electronic band structure. When the sample is annealed at high temperature, several processes such as recrystallization, oxidation, migration of vacancies, and changes in the surface structure are possible in the material which could generate the defects responsible for the atomiclike emission lines. Recent theoretical results from the firstprinciples calculations based on hybrid density functional by Mackoit-Sinkevičiene et al. [23] showed that the $4.1 \mathrm{eV}$ luminescence peak could be caused by the carbon dimer defect $\left(\mathrm{C}_{\mathrm{B}} \mathrm{C}_{\mathrm{N}}\right)$, which is a complex formed by two impurity carbon atoms on nearest-neighbor sites in the h-BN lattice. Another theoretical study using quantum chemistry calculations on $\mathrm{h}-\mathrm{BN}$ monolayer suggested that carbon clusters from two to four atoms such as $\mathrm{C}_{\mathrm{B}} \mathrm{C}_{\mathrm{N}}, \mathrm{C}_{4 \text { cis }}$, and $\mathrm{C}_{4}$ trans defects give luminescence in the range of 3.9 to $4.8 \mathrm{eV}$ [24]. Thus, the sharp emission lines observed in the UV region could be related to carbon defects which are generated during the annealing process. Carbon-related defect is also proposed as the source of emitters in the visible spectral range [25].

\section{Conclusions}

We studied luminescence properties of annealed h-BN crystal powders by using a deep UV PL spectroscopy. The h-BN samples annealed around $700{ }^{\circ} \mathrm{C}$ for $1 \mathrm{~h}$ in ambient air showed strong PL intensity at $5.49 \mathrm{eV}$. Additionally, the sample exhibited sharp atomic-like emission lines in UV region at 4.10, 4.12, 4.14, and $4.16 \mathrm{eV}$. Power- and temperature-dependent PL measurements of the sharp atomic-like emission lines exhibited robust nature of the energy peak positions. Due to the lack of correlation measurements, we are not sure if they are quantum emitters, but the small line width strongly indicates quantum emitter. Based on the theoretical reports, the sharp emission lines could be carbonrelated complex defects.
Acknowledgments This work was partially supported by PSC-CUNY Grant.

\section{References}

1. K. Watanabe, T. Taniguchi, H. Kanda, Nat. Mater. 3, 404 (2004)

2. L. Museur, D. Anglos, J.P. Petitet, J.P. Michel, A.V. Kanaev, J. Lumin. 127(2), 595 (2007)

3. L. Museur, E. Feldbach, A. Kanaev, Phys. Rev. B 78(15), 155204 (2008)

4. S. Majety, X.K. Cao, J. Li, R. Dahal, J.Y. Lin, H.X. Jiang, Appl. Phys. Lett. 101, 051110 (2012)

5. G. Cassabois, P. Valvin, B. Gil, Nat. Photonics 10, 262 (2016)

6. A. Bergh, G. Craford, A. Duggal, R. Haitz, Phys. Today 54(December), 42 (2001)

7. A. Khan, K. Balakrishnan, T. Katona, Nat. Photonics 2, 77 (2008)

8. I. Aharonovich, D. Englund, M. Toth, Nat. Photonics 10, 631 (2016)

9. T.T. Tran, C. Elbadawi, D. Totonjian, C.J. Lobo, G. Grosso, H. Moon, D.R. Englund, M.J. Ford, I. Aharonovich, M. Toth, ACS Nano 10, 7331 (2016)

10. N.R. Jungwirth, B. Calderon, Y. Ji, M.G. Spencer, M.E. Flatté, G.D. Fuchs, Nano Lett. 16, 6052 (2016)

11. T.Q.P. Vuong, G. Cassabois, P. Valvin, E. Rousseau, A. Summerfield, C.J. Mellor, Y. Cho, T.S. Cheng, J.D. Albar, L. Eaves, C.T. Foxon, 2D Mater. 4, 021023 (2017)

12. A. Hernández-Mínguez, J. Lähnemann, S. Nakhaie, J.M.J. Lopes, P.V. Santos, Phys. Rev. Appl. 10, 044031 (2018)

13. R. Bourrellier, S. Meuret, A. Tararan, O. Stéphan, M. Kociak, L.H. Tizei, A. Zobelli, Nano Lett. 16, 4317 (2016)

14. N. Maharjan, P. Joshi, R.C. Rai, M.L. Nakarmi, J. Lumin. 234, $117944(2021)$

15. D. Ganta, G. Venugopal, A.T. Hunt, M. Sapp, ISRN Nanotechnol. (2012). https://doi.org/10.5402/2012/608756

16. N. Maharjan, R.C. Rai, D.D. Mulmi, M.L. Nakarmi, J. Lumin. 219, 116879 (2020)

17. R. Bourrellier, M. Amato, L.H. Galvão Tizei, C. Giorgetti, A. Gloter, M.I. Heggie, K. March, O. Stephan, L. Reining, M. Kociak, A. Zobelli, ACS Photonics 1, 857 (2014)

18. L. Weston, D. Wickramaratne, M. Mackoit, A. Alkauskas, C.G. Van de Walle, Phys. Rev. 97, 214104 (2018)

19. J. Serrano, A. Bosak, R. Arenal, M. Krisch, K. Watanabe, T. Taniguchi, H. Kanda, A. Rubio, L. Wirtz, Phys. Rev. Lett. 98, 095503 (2007)

20. T. Pelini, C. Elias, R. Page, L. Xue, S. Liu, J. Li, J.H. Edgar, A. Dréau, V. Jacques, P. Valvin, B. Gil, Phys. Rev. Mater. 3(9), 094001 (2019)

21. I. Pamkove, Optical Processes in Semiconductors (Dover Publications, Inc., New York, 1971), pp. 132-142

22. G. Cassabois, P. Valvin, B. Gil, Phys. Rev. B 93, 035207 (2016)

23. M. Mackoit-Sinkevičienè, M. Maciaszek, C.G. Van de Walle, A. Alkauskas, Appl. Phys. Lett. 115, 212101 (2019)

24. T. Korona, M. Chojecki, Int. J. Quantum Chem. 119, e25925 (2019)

25. N. Mendelson, D. Chugh, J.R. Reimers, T.S. Cheng, A. Gottscholl, H. Long, C.J. Mellor, A. Zettl, V. Dyakonov, P.H. Beton, S.V. Novikov, Nat. Mater. 20(3), 321-328 (2021) 